\title{
Interactions: understanding people and process in prescribing in primary care
}

\author{
Carol Sinnott
}

\section{Correspondence to}

Dr Carol Sinnott, Department of Public Health and Primary Care, School of Clinical Medicine, University of Cambridge, Cambridge CB2 OSR, UK; cs926@medschl.cam.ac.uk

Accepted 1 December 2017 Published Online First 26 December 2017

\section{SLinked}

http://dx.doi.org/10.1136/ bmjqs-2017-006917

Check for updates

To cite: Sinnott $C$. BMJ Qual Saf 2018:27:176-178.
Internationally, primary care is under pressure. In 10 developed nations surveyed for the 2015 Commonwealth report, primary care physicians reported their struggle to find ways to care for ageing populations with complex healthcare needs. ${ }^{1}$ Practice consultation rates, average consultation duration and total patient-facing clinical workload have increased substantially in primary care in recent years. ${ }^{2}$ Increases in demand have not been matched by growth in either funding or in workforce: in the UK alone, the shortage of general practitioners (GPs) is expected to worsen from 3300 in 2015 to 8000 by 2020 . Over and above rising demand, factors such as advances in technology, the shifting workload from secondary to primary care, and patients' growing health and social care needs (including more complicated drug regimens and challenging national clinical standards) have all added to complexity. ${ }^{3}$

Much discussion has focused on how to increase the supply of primary care physicians. But, despite rapidly accelerating changes in the composition of the primary care workforce and its practices, far less attention has been given to understanding the teams that support physicians in primary care and how these individuals work together. Grant and Guthrie ${ }^{4}$ draw back the veil in an intriguing ethnographic study that shows how eight heterogeneous primary care practices in England and Scotland implement team approaches to the work of prescribing.

Prescribing in primary care constitutes a high-volume activity, and one that is vulnerable to error. In the UK, over one billion prescription items are dispensed in the community per year, equating to 2.7 million a day, or over 1900 a minute. ${ }^{5}$ A 2012 study commissioned by the UK General Medical Council found that $5 \%$ of prescription items were associated with a prescribing error, and around 1 in 550 prescription items contained a severe error. ${ }^{6}$ Strong internal processes are needed to ensure safe prescribing. Defences against prescribing errors may be employed at the level of the prescriber, practice-wide or through health information technology. ${ }^{67}$ Many interventions to improve prescribing safety have focused on upskilling physicians in therapeutics and pharmacology, ${ }^{89}$ introducing pharmacists into practices ${ }^{10}$ and using computer decision support. ${ }^{11}$

An important achievement of Grant and Guthrie is in offering one of the first detailed accounts of the role of non-clinical practice staff in the processing of prescriptions, and of how the 'work' of prescribing happens across a practice. Their ethnographic observations (2000 hours of them, along with 62 semistructured interviews) captured administrative work, corridor conversations, GP work during coffee breaks, allocation of tasks and back-office conversations. By observing and interviewing all staff involved in the generation of a signed authorised prescription, the researchers discovered the shared understandings and behaviours necessary to function within these teams.

One of their key insights is into the tradeoffs made by practices in the handling of high volumes of prescription requests (up to 2000 prescriptions per week). The practices variably prioritised speed of processing (efficiency) over allowing time for clinical judgement (thoroughness) in the generation of prescriptions. Examining the work allocated to receptionists revealed how practices balanced these imperatives. In all cases, receptionists filtered prescription requests and identified which they could issue and print themselves, and which would require 
physician review. Efficiency-focused practices gave greater authority to receptionists to issue, amend or print repeat and non-repeat prescriptions, and justified this autonomy by the receptionists' extensive knowledge of the patients, gained from many years of working in the practice. Thoroughness-focused practices adopted a more restrictive approach where receptionists issued only a limited range of prescription items or items previously authorised by a GP. Although the practices that did this considered this approach safer, it led to greater numbers of prescription requests being directed to GPs for attention, additional time required to process requests and greater impact on GPs' already saturated schedules. Thus, prioritising thoroughness over speed of processing was not free of risk either.

Grant and Guthrie highlight how prescribing processes in primary care rely on interdisciplinary work, interpersonal relationships and non-clinical expertise that may be gained over years. These findings echo those of Swinglehurst et al, ${ }^{7}$ who previously described the crucial contributions made by receptionists as they try to bridge the gap between formal practice protocols and repeat prescribing as it plays out in real life. Their 'hidden' contributions are essential to getting the job done and contribute in indirect ways to maintaining safety by ensuring timely issue of medications. Efforts to learn from receptionists' first-hand knowledge of the reality of the system at the front line could make an invaluable contribution to safety.

In many ways, these findings do not come as a surprise: they are consistent with the evidence on soft intelligence, which recognises the value of the collective knowledge and experience of front-line staff in driving sustainable change and service improvement. ${ }^{12}$ The broader achievement of this study then is to encourage a move away from the traditional view of organisational culture in primary care as hierarchical and physician-centred to one that is interdisciplinary and reliant on team dynamics. ${ }^{13} 14$

National policies on primary care, seeking to address both escalating costs and physician shortages, increasingly emphasise the need for greater delegation of tasks that can be safely and competently managed by others. ${ }^{15}$ Recognising the contribution of non-clinical staff and ensuring they feel that their work is important and their performance visible to other team members will help to build a collaborative culture in primary care practices, with potential benefits in improved care access and continuity, better chronic disease control, and increased patient satisfaction. ${ }^{16}$ Greater team cohesion in primary care can also reduce duplication, streamline patient care and enable specialist skills to be used more cost-effectively. ${ }^{17}$ This requires practices to ensure the goals, responsibilities and insights of all team members are understood and valued by all. ${ }^{18}$

The issuing of prescriptions in primary care has been shown to be a complex, technology-supported process that requires collaboration between clinical and administrative staff. As policy urges primary care physicians to stretch safe delegation further, quality improvement research in primary care must also take a team or organisational perspective. There is a need for quality improvement efforts to understand the team structure, role definitions and accountability. This will better identify how the team can work to maintain and promote safety, and identify points where improvement is possible.

Funding CS is supported by a National Institute for Health Research Clinical Lectureship.

Competing interests None declared.

Provenance and peer review Commissioned; internally peer reviewed.

(C) Article author(s) (or their employer(s) unless otherwise stated in the text of the article) 2018. All rights reserved. No commercial use is permitted unless otherwise expressly granted.

\section{REFERENCES}

1 Osborn R, Moulds D, Schneider EC, et al. Primary care physicians in ten countries report challenges caring for patients with complex health needs. Health Aff 2015;34:2104-12.

2 Baird B, Charles A, Honeyman M, et al. Understanding pressures in general practice. The Kings Fund, 2016.

3 NHS National Patient Safety Agency. Seven steps to patient safety for primary care. London, 2006.

4 Grant S, Guthrie B. Efficiency and thoroughness trade-offs in high-volume organisational routines: an ethnographic study of prescribing safety in primary care. BMJ Qual Saf 2018;27:199-206.

5 Health and Social Care Information Centre, NHS Digital. More than 1 billion prescription items dispensed in a year - or 1,900 a minute. http://content.digital.nhs.uk/article/3199/ More-than-1-billion-prescription-items-dispensed-in-a-year-or1900-a-minute (accessed 12 Oct 2017).

6 Avery A, Barber N, Ghaleb M, et al. Investigating the prevalence and causes of prescribing errors in general practice: the PRACtICe study: General Medical Council, 2012.

7 Swinglehurst D, Greenhalgh T, Russell J, et al. Receptionist input to quality and safety in repeat prescribing in UK general practice: ethnographic case study. BMJ 2011;343:d6788.

8 Arnold SR, Straus SE. Interventions to improve antibiotic prescribing practices in ambulatory care. Cochrane Database Syst Rev 2005;4:CD003539.

9 Kamarudin G, Penm J, Chaar B, et al. Educational interventions to improve prescribing competency: a systematic review. BMJ Open 2013;3:e003291.

10 Patterson SM, Cadogan CA, Kerse N, et al. Interventions to improve the appropriate use of polypharmacy for older people. Cochrane Database Syst Rev 2014;10:CD008165.

11 Fraccaro P, Arguello Casteleiro M, Ainsworth J, et al. Adoption of clinical decision support in multimorbidity: a systematic review. JMIR Med Inform 2015;3:e4.

12 Martin GP, McKee L, Dixon-Woods M. Beyond metrics? Utilizing 'soft intelligence' for healthcare quality and safety. Soc Sci Med 2015;142:19-26.

13 Lawrence M, Packwood T. Adapting total quality management for general practice: evaluation of a programme. Qual Health Care 1996;5:151-8. 


\section{Editorial}

14 Geboers H, van der Horst M, Mokkink H, et al. Setting up improvement projects in small scale primary care practices: feasibility of a model for continuous quality improvement. Qual Health Care 1999;8:36-42.

15 NHS England: General Practice Forward View. 2016 https:// www.england.nhs.uk/wp-content/uploads/2016/04/gpfv.pdf

16 Soubhi H, Bayliss EA, Fortin M, et al. Learning and caring in communities of practice: using relationships and collective learning to improve primary care for patients with multimorbidity. Ann Fam Med 2010;8:170-7.

17 Ross F, Rink E, Furne A. Integration or pragmatic coalition? An evaluation of nursing teams in primary care. J Interprof Care 2000;14:259-67.

18 Borrill CCJ, Carter A, Dawson J, et al. The effectiveness of health care teams in the national health service. Birmingham: University of Aston, 2000. 